PROCEEDINGS OF THE

AMERICAN MATHEMATICAL SOCIETY

Volume 133, Number 2, Pages 357-362

S 0002-9939(04)07455-6

Article electronically published on September 16, 2004

\title{
ISOTRIVIALLY FIBRED ISLES IN THE MODULI SPACE OF SURFACES OF GENERAL TYPE
}

\author{
MARTIN MÖLLER \\ (Communicated by Michael Stillman)
}

\begin{abstract}
We complement Catanese's results on isotrivially fibred surfaces by completely describing the components containing an isotrivial surface with monodromy group $\mathbb{Z} / 2 \mathbb{Z}$. We also give an example for deformation equivalent isotrivial surfaces with different monodromy group.
\end{abstract}

\section{INTRODUCTION}

In Ca00, Catanese introduced the notion of 'surfaces isogenous to a product', partly because the notion of 'isotrivially fibred surface' is not stable under deformation. For the former he obtains a complete description of the moduli space (of surfaces of general type) by means of fixed loci in products of Teichmüller spaces, while for isotrivially fibred surfaces his description is restricted to the sublocus of surfaces having the same topological type as a given isotrivially fibred surface minus their singular fibres.

In this paper we complement these results in two ways. First we describe entirely the connected components $N_{2}$ of the moduli space, which contain these isotrivial isles in the simplest case of the monodromy group $G=\mathbb{Z} / 2 \mathbb{Z}$. We use the fact (Theorem 3.1) that these surfaces are double coverings of a product of curves if we impose some restrictions on the irregularity. This enables us to enumerate the connected components of $N_{2}$, and to calculate its dimension and that of its isotrivially fibred subloci (Corollaries 3.3 and 3.4).

Secondly we give an example, that this method does not extend to bigger monodromy groups, by showing that isotrivially fibred surfaces with different monodromy groups can occur in one connected component of the moduli space (Proposition 4.1). For this purpose we use the description of abelian coverings given in [FP97.

The results of this paper are part of the author's dissertation ([Mo02]), which describes moduli spaces for different types of fibred surfaces, by exploiting high irregularity.

\section{Notation.}

- We use the complex numbers $\mathbb{C}$ as our base field throughout.

Received by the editors December 12, 2002 and, in revised form, July 7, 2003.

2000 Mathematics Subject Classification. Primary 14J10, 14D06.

Key words and phrases. Isotrivially fibred surfaces, monodromy group.

(C)2004 American Mathematical Society 
- By a surface of general type $X$ we think of its canonical model, i.e. a normal surface with $K_{X}$ ample and at most rational double points.

- $\mathfrak{S}(\cdot)$ is the functor that associates to a scheme $T$ the isomorphism classes of families of surfaces of general type over $T$. 'Family' always implies proper and flat. We denote by $N$ its coarse moduli space.

- A fibration of $X$ is a morphism $X \rightarrow B$ to a smooth curve $B$ of genus $b$ with connected fibres. Let $g$ denote the genus of the fibres. We call $(g, b)$ the type of the fibration. We will suppose that $g \geq 2$ (fibre condition $(F C)$ ) and $b \geq 2$ (base condition $(B C)$ ), so that by [Be91] and [Ca00 the property of having a type $(g, b)$-fibration is deformation invariant. This also implies that minimal models and relatively minimal models coincide, and $X$ admits such a fibration if and only if the minimal model does.

- We denote by $\mathfrak{S}_{g, b}(\cdot)$ the subfunctor of $\mathfrak{S}(\cdot)$ parametrizing $(g, b)$-fibred surfaces. We only employ this notation when $(B C)$ and $(F C)$ are fulfilled.

- We use different notations to distinguish between a line bundle $L$ on a surface $X \in \mathfrak{S}(\operatorname{Spec} \mathbb{C})$ and a family of line bundles $\mathcal{L}$ on a family of surfaces $X \rightarrow T$. Given a locally free sheaf $L$ on $X$, we denote the associated geometric vector bundle by $\mathbb{V}(L) \rightarrow X$.

\section{Monodromy and Albanese-Image}

Given a fibration $h: X \rightarrow B$, we let $B^{\prime}$ be the locus where the fibres are smooth. Recall that $h$ is said to be isotrivial (or of constant moduli) if the image of the canonical mapping $B^{\prime} \rightarrow M_{b}$ to the moduli space for smooth curves is just one point.

Due to (FC) there is a group $G$, the monodromy group, such that after a Galois base change $C_{1} \rightarrow B$ with group $G$, étale outside the images of degenerate fibres, we have

$$
C_{1} \times C_{2} \cong C_{1} \times_{B} X
$$

where $C_{2}$ is a fibre of $h . G$ acts on both $C_{1}$ and $C_{2}$, and we denote the quotient under the diagonal action by $Q=\left(C_{1} \times C_{2}\right) / G$.

For symmetry let $B=B_{1}, b=b_{1}, B_{2}=C_{2} / G$ and $g\left(B_{2}\right)=b_{2}$. We abbreviate $Y=B_{1} \times B_{2}$ and call the projections $p_{i}: Y \rightarrow B_{i}$. By [Ser96] we know that $q(X)=b_{1}+b_{2}$, and if $b_{2}>0$, the natural birational map $\varepsilon: X \rightarrow Q$ is a morphism, i.e. $X$ has two fibrations $h_{1}: X \rightarrow B_{1}$ and $h_{2}: X \rightarrow B_{2}$.

Details for this can be found in [Ser96] and [Ca00].

Lemma 2.1. Let $X \rightarrow T \in \mathfrak{S}_{g, b}$ be a family of surfaces. Suppose that (at least) one fibre admits an is isotrivial fibration with $q \geq b+2$. If $X \rightarrow T$ admits a section, the image of the (relative) Albanese-map $\alpha$ is a family of products of smooth curves $B_{i}(i=1,2)$ over $T$ of genera $b_{1}$ and $b_{2}$.

Proof. Suppose $T=\operatorname{Spec} \mathbb{C}$ and $X$ is isotrivial. The Albanese variety has dimension $b_{1}+b_{2}$, and its universal property applied to $h_{1} \times h_{2}$ induces an isogeny $\operatorname{Alb}(X) \rightarrow$ $\operatorname{Jac}\left(B_{1}\right) \times \operatorname{Jac}\left(B_{2}\right)$ and hence a finite morphism $\alpha(X) \rightarrow Y$. The image of $Y$ under the inverse isogeny generates the Albanese variety. Since we excluded the cases $b_{1}=1$ and $b_{2}=1$, this is only possible when $\alpha(X)=Y$.

This argument did not make full use of the isotriviality but only of the existence of both fibrations and numerical conditions, i.e. deformation invariant properties. 
Hence it also applies to the other fibres and in the relative setting by rigidity of a product of curves.

Remark 2.2. If $q=b_{1}+1$, the Albanese image need not be a product of $B_{1}$ and an elliptic curve. This is the reason why we exclude this case, although similar investigations can be made also for coverings of elliptic fibrations.

\section{ISOTRIVIAL SURFACES WITH MONODROMY GROUP $G=\mathbb{Z} / 2 \mathbb{Z}$}

We restrict ourselves in this section to surfaces in $\mathfrak{S}_{g, b}^{2}$, which is the subfunctor of $\mathfrak{S}_{g, b}$ with $q \geq b_{1}+2$ parametrizing surfaces, whose (generic) degree of the Albanese map equals 2 and where the corresponding components of the moduli space contain an isotrivially fibred surface, i.e. such that Lemma 2.1 applies. We shall exclude the case that $G$ acts freely on $C_{1} \times C_{2}$, which is dealt with in Ca00] (those surfaces will be called isogenous to a product).

Under these conditions Lemma 2.1 ensures for each $X \in \mathfrak{S}_{g, b}^{2}(T)$ the existence of fibrations $h_{i}: X \rightarrow B_{i}$ : The section required there can be created after an étale base change and these fibrations (though not the Albanese morphism) descend to $T$. The next theorem will show that $\alpha$ is not only generically of degree 2 but in fact finite.

Recall that a double covering $X \rightarrow Y$ of surfaces over $T$ is given by data $(L, D)$, where $D$ is an effective, flat divisor on $Y$ and $L$ is a line bundle on $Y$ with $L^{\otimes 2}=$ $\mathcal{O}_{Y}(D)$.

Theorem 3.1. The surfaces in $\mathfrak{S}_{g, b}^{2}(\mathbb{C})$ are double coverings of a product of curves $Y=B_{1} \times B_{2}$ of genus $b_{i}, b_{i} \geq 2$, ramified over a curve with at most simple singularities.

A general surface in $\mathfrak{S}_{g, b}^{2}(\mathbb{C})$ is smooth, i.e. given $X_{0} \in \mathfrak{S}_{g, b}^{2}(\mathbb{C})$, there is a family $X \rightarrow T \in \mathfrak{S}_{g, b}^{2}(T)$ over a 1-dimensional pointed base $(T, 0)$, whose fibre over 0 is $X_{0}$ and where all the other fibres are smooth.

A surface $X$ in $\mathfrak{S}_{g, b}^{2}(\mathbb{C})$ is isotrivially fibred if and only if the branch divisor of $X \rightarrow Y$ is composed of horizontal and vertical curves.

Proof. Let $X \in \mathfrak{S}_{g, b}^{2}(\mathbb{C})$. Stein factorisation gives $X \rightarrow X^{\prime} \rightarrow Y$, where $X^{\prime}$ is a double covering of $Y$. $X^{\prime}$ has only rational double points (and hence coincides with $X$ ) if and only if $D$ has only simple singularities ([BPV84] Theorem II.5.1). Thus we have to show that the open subfunctor

$$
\widetilde{\mathfrak{S}_{g, b}^{2}}(T)=\left\{X \rightarrow T \in \mathfrak{S}_{g, b}^{2}(T) \mid X \rightarrow \alpha(X) \text { is finite }\right\}
$$

is also closed. Let $X \rightarrow T \in \mathfrak{S}_{g, b}^{2}(T)$ be a family over the pointed scheme $(T, 0)$, such that the restriction to $T^{\prime}=T \backslash 0$ is in $\widetilde{\mathfrak{S}_{g, b}^{2}}\left(T^{\prime}\right)$. After an étale base change we may suppose that $X$ admits a simultaneous resolution of singularities. $\left.X\right|_{T^{\prime}}$ comes with an involution $\tau$ whose quotient is $\left.\alpha(X)\right|_{T^{\prime}}$. Since $X$ has an ample canonical divisor, we can apply [FP97] Prop. 4.4 to extend $\tau$ to an involution on $X$. All we need is to show that for the fibre over 0 we have $X_{0} / \tau \cong \alpha\left(X_{0}\right)$. But this follows noting that $\alpha$ factors via $X / \tau$ and that $X / \tau \rightarrow T$ is flat (see e.g. [Ma97]).

The second assertion follows from the first and the theorem of Bertini, once we have shown that $L$ is ample. This immediately follows from the exclusion of the case of surfaces isogenous to a product, and the last assertion should be clear by the given description. 
Lemma 3.2. If $X \rightarrow T \in \mathfrak{S}_{g, b}^{2}(T)$ admits a section, there are line bundles $\mathcal{L}_{i}$ on $B_{i}$, such that $\mathcal{L}=p_{1}^{*} \mathcal{L}_{1} \otimes p_{2}^{*} \mathcal{L}_{2}$.

Proof. Due to the section we have

$$
\mathrm{Pic}_{B_{1} \times B_{2}} / T \cong \mathrm{Pic}_{B_{1} / T} \times \operatorname{Pic}_{B_{2} / T} \times \operatorname{Corr}\left(B_{1}, B_{2}\right),
$$

where $\operatorname{Corr}\left(B_{1}, B_{2}\right)$ is the functor of divisorial correspondences between $B_{1}$ and $B_{2}$. If $T=\operatorname{Spec} \mathbb{C}$ and $X$ is isotrivially fibred, we know that $L^{2}=\mathcal{O}_{Y}(D)=$ $p_{1}^{*} \mathcal{O}_{B_{1}}\left(D_{1}\right) \otimes p_{2}^{*} \mathcal{O}_{B_{2}}\left(D_{2}\right)$. Since $\operatorname{Corr}\left(B_{1}, B_{2}\right)$ has no nontrivial nilpotent elements, this splitting is also possible for $L$, and since the zero section of $\operatorname{Corr}\left(B_{1}, B_{2}\right)$ is an open and closed immersion, this remains also valid for arbitrary $X \rightarrow T \in$ $\mathfrak{S}_{g, b}^{2}(T)$.

By an étale base change we can always suppose that a section exists. To analyse dimensions and connected components of the moduli space, we can hence use in the sequel the functor

$$
Q(T)=\left\{\left(Y / T, \mathcal{L}_{1}, \mathcal{L}_{2}, s\right)\right\}
$$

where

$$
Y=B_{1} \times B_{2} \in \mathfrak{C}_{b_{1}}(T) \times \mathfrak{C}_{b_{2}}(T), \mathcal{L}_{i} \in \operatorname{Pic}_{B_{i} / T}, s \in \Gamma\left(Y, p_{1}^{*} \mathcal{L}_{1} \otimes p_{2}^{*} \mathcal{L}_{2}\right)
$$

instead of $\mathfrak{S}_{g_{1}, b_{1}}^{2}(T)$.

If we let $d_{i}=\operatorname{deg} L_{i}$, our description yields:

Corollary 3.3. Two surfaces in $\mathfrak{S}_{g_{1}, b_{1}}^{2}(\mathbb{C})$ lie in the same connected components if and only if their invariants $b_{1}, b_{2}, d_{1}$ and $d_{2}$ coincide. We hence denote these components by $N_{2}\left(b_{1}, b_{2}, d_{1}, d_{2}\right)$.

Proof. By the above theorem the conditions are clearly necessary.

Let $y=\mathcal{B}_{1} \times \mathcal{B}_{2} \rightarrow M^{[n]}$ denote the universal family of products of smooth curves together with level- $n$-structures (to obtain a fine moduli space). Fix a line bundle $L=h_{1}^{*} L_{1} \otimes h_{2}^{*} L_{2}$ on $Y$, and let $g: P=\operatorname{Pic}_{y / M^{[n]}}^{L} \rightarrow M^{[n]}$ be the component of the relative Picard scheme parametrizing line bundles linearly equivalent to $L$. Next we take the scheme $F$ representing the functor

$$
T \mapsto\left\{(y, \mathcal{L}, s) \mid(y, \mathcal{L}) \in h_{P}(T), s \in \Gamma(y, \mathcal{L})\right\},
$$

where $h_{P}$ denotes the functor of points of $P$. The existence of $F$ is guaranteed by (EGA III, Theorem 7.7.6) and (EGA I, Prop. 9.4.9). We will restrict $F$ to the open locus $F^{\prime}$, which parametrizes sections $s$ whose zero locus has only simple singularities, and we denote by $f$ the natural morphism $F \rightarrow P$. The fibres of $f$ are $H^{0}\left(C_{1}, L_{1}\right) \otimes H^{0}\left(C_{2}, L_{2}\right)$, where $C_{i}$ and $L_{i}$ are the curves and bundles corresponding to the image. Of course, if this vector space is non-zero, the intersection with $F^{\prime}$ is dense in it.

We claim that $f\left(F^{\prime}\right)$ is connected: This is the locus of quadruples $\left(C_{i}, L_{i}\right)(i=$ $1,2)$ such that $H^{0}\left(C_{1}, L_{1}\right) \otimes H^{0}\left(C_{2}, L_{2}\right)$ is non-zero. $g$ maps $f\left(F^{\prime}\right)$ properly onto $M^{[n]}$, which is connected. The fibre of $g$ over $C_{1} \times C_{2}$ is $W_{d_{1}}^{0}\left(C_{1}\right) \times W_{d_{2}}^{0}\left(C_{2}\right)$, using the notation in ACGH85]. This space is connected by [ACGH85, Theorem V.1.4, and our claim follows by elementary topology.

Suppose $F^{\prime}$ is the disjoint union of closed subsets $A$ and $B$. Then $\overline{f(A)}$ and $\overline{f(B)}$ have a common point $p \in f\left(F^{\prime}\right)$. Suppose $p \in f(A)$, and hence $f^{-1}(p) \subset A$. Let $B_{0} \subset f(B)$ be a subset, over which the fibres of $f$ have constant positive dimension (i.e. $\left.f\right|_{B_{0}}$ is a bundle of vector spaces) and such that $p \in \overline{B_{0}}$. Consider the closure 
$C=\overline{f^{-1}\left(B_{0}\right)}$ in $F$, which is still a bundle of vector spaces and whose image under $f$ contains p. $C \cap F^{\prime}$ is dense in each fibre of $\left.f\right|_{C}$, and by construction $B \cap C$ is dense in $C$. This implies that $f^{-1}(p) \cap B \neq \emptyset$, a contradiction.

For large $d_{i}$ we can easily compute the dimension of the moduli space and the locus. Let $h_{i}^{0}=\operatorname{dim} H^{0}\left(B_{i}, L_{i}^{\otimes 2}\right)$ for $i=1,2$.

Corollary 3.4. If $2 d_{i}>2 b_{i}-2$ (i.e $L^{\otimes 2}-K_{Y}$ is ample), the dimension of $N_{2}\left(b_{1}, b_{2}, d_{1}, d_{2}\right)$ is

$$
\operatorname{dim} N_{2}=4 b_{1}+4 b_{2}-7+h_{1}^{0} h_{2}^{0} .
$$

Isotrivial surfaces form irreducible, closed subvarieties in $N_{2}\left(b_{1}, b_{2}, d_{1}, d_{2}\right)$ of dimension $4 b_{1}+4 b_{2}+h_{1}^{0}$ and of dimension $4 b_{1}+4 b_{2}+h_{2}^{0}$.

Proof. $N_{2}\left(b_{1}, b_{2}, d_{1}, d_{2}\right)$ has a canonical morphism to the moduli space of pairs of curves together with a line bundle of degrees $d_{1}$ and $d_{2}$ respectively. This space is irreducible and has dimension $4 b_{1}+4 b_{2}-6$. The ampleness of $L^{\otimes 2}-K_{Y}$ ensures that the fibres are dense in a vector space of dimension $h_{1}^{0} h_{2}^{0}-1$, and this gives the first assertion.

We obtain the irreducible components of the locus of isotrivial surfaces by taking only sections of the form $s=s^{1} \otimes s^{2}$, i.e. by fixing $s^{1}$ and letting $s^{2}$ vary or vice versa. The degree hypothesis implies that there are no obstructions to deforming these sections.

Remark 3.5. These results illustrate in the 'simplest possible' case Catanese's result (Ca00] Theorem 5.4) that if we fix the 'topological type' (see loc. cit. for details) of the isotrivially fibred surface minus the singular fibres, we obtain irreducible components of the isotrivial locus,

\section{THE MONODROMY GROUP IS NOT INVARIANT UNDER DEFORMATIONS}

This section contains the 'negative' result that in general one cannot hope to analyse components of the moduli space containing isotrivial surfaces by fixing the monodromy group.

Proposition 4.1. If the isotrivial surfaces $X_{1}$ and $X_{2}$ belong to one connected component of $N_{g, b}$, the orders of the respective monodromy groups $G_{1}$ and $G_{2}$ are equal, but these groups need not be isomorphic.

Proof. The first statement is clear because of Lemma 2.1 and the fact that the group order is the (generic) degree of the Albanese map.

To prove the second we can take the simplest possible case $G_{1}=\mathbb{Z} / 4 \mathbb{Z}$ and $G_{2}=(\mathbb{Z} / 2 \mathbb{Z})^{2}$ and use the description of the isotrivial surfaces as abelian coverings of $Y=B_{1} \times B_{2}$. Fix $Y$ with $b_{i} \geq 2$, and take line bundles $L_{i}$ on $B_{i}$ together with sections $s^{i} \in \Gamma\left(B_{i}, L_{i}^{\otimes 4}\right)$. Let $L=p_{1}^{*} L_{1} \otimes p_{2}^{*} L_{2}$ and $s=s^{1} \otimes s^{2}$. The surface

$$
X_{G_{1}}=V\left(w_{1}^{4}-s\right) \subset V\left(L^{\otimes 4}\right),
$$

where $w_{1}$ is a local coordinate of $L$ and $V$ denotes the vanishing locus, is an isotrivial $G_{1}$-covering of $Y$. Since $X_{G_{1}}=\left(C_{1} \times C_{2}\right) / G_{1}$, where $C_{i}$ is the $G_{1}$-covering corresponding to $L_{i}$ and $s^{i}$, this surface has at most $A_{3}$ singularities. Due to the conditions on $b_{i}$, no rational curve is contained in $X_{G_{1}}$, which is hence in $\mathfrak{S}_{g, b}$ (where, as usual, $b=b_{1}$ and $g$ is the genus of the fibres of $X \rightarrow B_{1}$ ). 
Fix two generators $\alpha$ and $\beta$ of $G_{2}$ and two sections $s_{\alpha}=s_{\alpha}^{1} \otimes s_{\alpha}^{2} \in \Gamma\left(Y, L^{\otimes 4}\right)$, $s_{\beta}=s_{\beta}^{1} \otimes s_{\beta}^{2} \in \Gamma\left(Y, L^{\otimes 2}\right)$. We can arrange that the zeros of $s_{\alpha}^{i}$ and $s_{\beta}^{i}(i=1,2)$ are disjoint. Hence the surface

$$
X_{G_{2}}=V\left(w_{\alpha}^{2}-s_{\alpha}, w_{\beta}^{2}-s_{\beta}\right) \subset \mathbb{V}\left(L^{\otimes 4} \oplus L^{\otimes 2}\right),
$$

where $w_{\alpha}$ and $w_{\beta}$ are local coordinates of $L^{\otimes 2}$ and $L$, has only $A_{1}$-singularities, i.e. is indeed in $\mathfrak{S}_{g, b}$.

We construct a surface $X_{1}$ that is easily seen to deform to both $X_{G_{1}}$ and $X_{G_{2}}$. Suppose there are $s_{2} \in \Gamma\left(Y, L^{\otimes 2}\right)$ and $s_{4} \in \Gamma(Y, L)$ such that $\operatorname{div}\left(s_{2}\right)$ and $\operatorname{div}\left(s_{4}\right)$ are both smooth and have only normal crossings and such that $\operatorname{div}\left(s_{4}+s_{2}^{2} / 4\right)$ is smooth. We claim that, denoting $w_{i}$ local coordinates of $L^{\otimes i}$,

$$
X_{1}=V\left(w_{2}^{2}-s_{2} w_{2}-s_{4}, w_{1}^{2}-w_{2}\right) \subset \mathbb{V}\left(L^{\otimes 4} \oplus L^{\otimes 2}\right)
$$

is in $\mathfrak{S}_{g, b}$ : The quotient $\tilde{X}_{1}$ of $X_{1}$ under $w_{1} \mapsto-w_{1}$ ramifies over $\operatorname{div}\left(s_{4}+s_{2}^{2} / 4\right)$ and is hence smooth. Also, $X_{1} \rightarrow \tilde{X}_{1}$ ramifies over $w_{2}=0$, which has smooth components and normal crossings by the hypotheses.

Obviously $X_{1}$ deforms to $V\left(w_{1}^{4}-s_{4}\right) \subset \mathbb{V}\left(L^{\otimes 4}\right)$, and this is a deformation of $X_{G_{1}}$. By change of coordinates $w_{\alpha}=w_{2}-s_{2} / 2, w_{1}=w_{\beta}$, we have

$$
X_{1} \cong V\left(w_{\alpha}^{2}-\left(s_{4}+s_{2}^{2} / 4\right), w_{\beta}^{2}-w_{\alpha}-s_{2} / 2\right) \subset \mathbb{V}\left(L^{\otimes 4} \oplus L^{\otimes 2}\right),
$$

which obviously deforms to $X_{G_{2}}$.

\section{ACKNOWLEDGEMENTS}

The author thanks his thesis advisor Frank Herrlich for his permanently open door to his office.

\section{REFERENCES}

[ACGH85] Arbarello, E., Cornalba, M., Griffiths, P.A., Harris, J., Geometry of Algebraic Curves, Volume I, Springer Grundlehren 267 (1985) MR0770932 (86h:14019)

[Be91] Beauville, A., Letter to F. Catanese, Appendix to [Ca91]

[BPV84] Barth, W., Peters, C., van de Ven, A., Compact Complex Surfaces, Ergebnisse der Math. 3 Bd. 4, Springer-Verlag (1984) MR0749574 (86c:32026)

[Ca91] Catanese, F., Moduli and classification of irregular Kaehler manifolds (and algebraic varieties) with Albanese general type fibrations, Invent. Math. 104 (1991), 263-289 MR:1098610|(92f:32049)

[Ca00] Catanese, F., Fibred surfaces, varieties isogenous to a product and related moduli spaces, Am. J. Math. 122 (2000), 1-44 MR.1737256 (2001i:14048)

[EGA] Grothendieck, A., Éléments de la géometrie algébrique I: Springer, Grundlehren 166 (1971) III: Publ Math. IHES 11 (1961) and 17 (1963)

[FP97] Fantechi, B., Pardini, R., Automorphisms and moduli spaces of varieties with ample canonical class via deformations of abelian covers, Comm. in Alg. 25(5) (1997), 14131441 MR1444010 (98c:14028)

[Ma97] Manetti, M., Iterated double covers and connected components of moduli spaces, Topology 36 No.3 (1997), 745-764 MR1422433 (98h:14045)

[Mo02] Möller, M., Modulräume irregulär gefaserter Flächen, Dissertation, Karlsruhe (2002)

[Ser96] Serrano, F., Isotrivial Fibred Surfaces, Annali di Matematica pura ed app. (IV), Vol. CLXXI (1996), 63-82 MR1441865 (98e:14036)

Mathematisches Institut II, Universität Karlsruhe, Englerstrasse 2 , D-76128 Karlsruhe, Germany

E-mail address: moeller@math.uni-karlsruhe.de

Current address: FB6 (Mathematik), Universität Essen, D-45117 Essen, Germany

E-mail address: martin.moeller@uni-essen.de 